\title{
II. THE GALAXY
}

(A) INTRODUGTION ON THE IMPORTANGE OF STELLAR EVOLUTION FOR PROBLEMS OF GALACTIG STRUGTURE

In connexion with the importance of present views on stellar evolution for discussions of the evolution of the Galaxy, the sessions dealing with galactic problems were preceded by a review of items relating the evolution of stars with that of the Galactic System. The following represents with only few modifications the introductory paper on this subject as presented by $\mathrm{J} . \mathrm{H}$. Oort.

Although we are still far from understanding how the galactic system has evolved, and how stars composing it have been formed, it seems that we have nevertheless approached a stage where it becomes useful to go beyond a simple description of stellar distribution and motions. Gradually some facts are emerging, which suggest at least a rudimentary understanding of why different objects are distributed in the way they are distributed.

A theory of the galactic system is primarily a theory of its evolution. Before considering this we summarize the principal facts concerning the differences between various constituents of the Galaxy. An extensive list of such differences has been given by Parenago at the Groningen conference in I953 [1]. A more limited compilation is presented by Table 2. The subdivisions and the names of the various groups are those advocated by the participants of the Semaine d'Etude on Stellar Populations (Rome, May 1957). For each population group rough indications are given of the average distance, $z$, in parsecs from the galactic plane; of the average speed, $Z$, in $\mathrm{km} / \mathrm{sec}$, in the direction perpendicular to the galactic plane; of the axis ratio of the sub-system; of the degree of concentration toward the galactic centre; and of the smoothness of the distribution. Next we give estimates of the heavy element abundance for each population group, of its age and of its total mass.

At the extreme left of Table 2 are the stars that populate the spherical sub-system, called the halo population II. Examples of objects belonging to this population are the sub-dwarfs, which probably form the most massive part of the population, the typical globular clusters, and the RR Lyrae variables with periods longer than $0 \cdot 4$ days approximately.

The second column represents an intermediate population II. The longperiod variables of early spectral classes and relatively short periods belong 


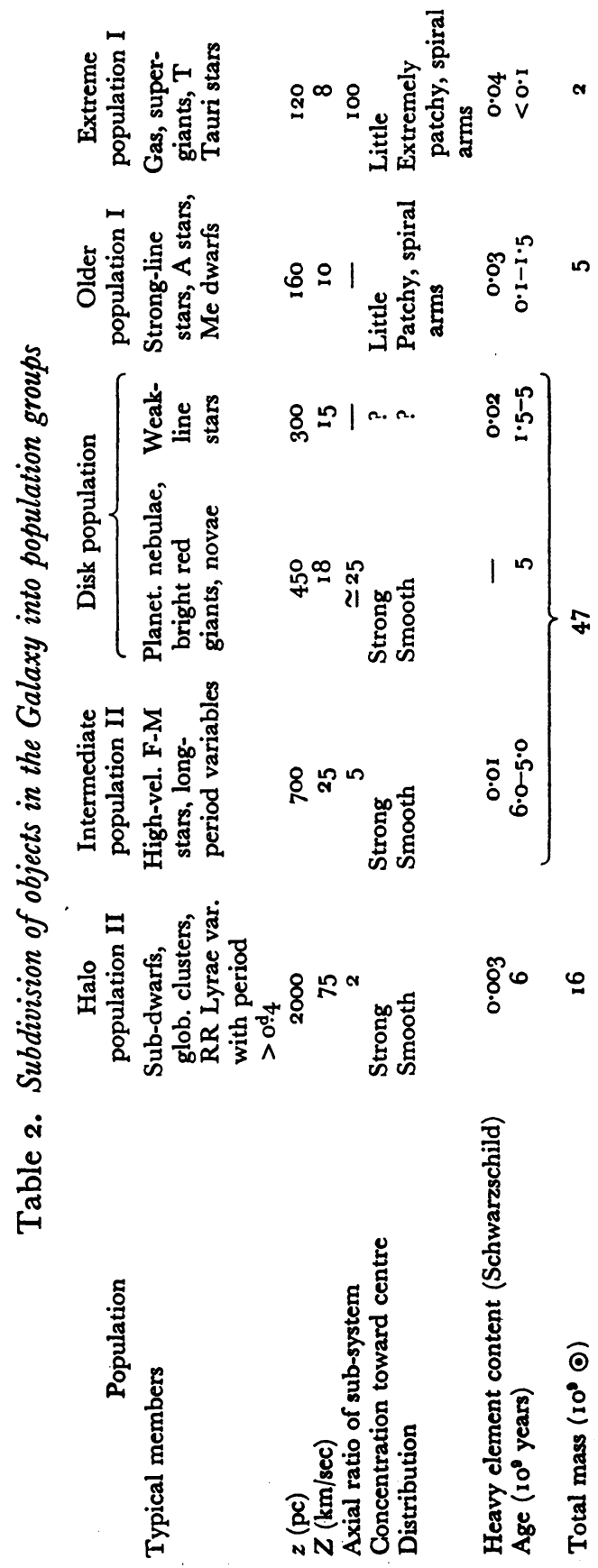

14 
at least partly to this intermediate population; also the ordinary highvelocity stars.

We next have the disk population, which has been divided over two columns in Table 2. Examples of this population are the planetary nebulae and novae, which have a rather strong concentration to the galactic plane. As another part of this disk population we consider the category of weakline stars of Miss Roman. While it is well known that the objects of the first three columns show strong concentration towards the galactic centre, nothing is known about the distribution of the weak-line stars in the plane of the Galaxy.

The intermediate population I or "Older Population I", like class A stars and the strong-line stars can be clearly distinguished from the rest. The same holds for the extreme population I, which includes among other objects the super-giants and the interstellar gas.

The estimates of the total mass of the various population groups, given in the bottom line of Table 2, are extremely uncertain and have been included only to give an indication of the relative mass distribution among the different components of the Galaxy. The most certain of these estimates is that of the extreme population $\mathrm{I}$, based on the $2 \mathrm{I} \mathrm{cm}$ measures of the interstellar hydrogen. For the intermediate population $I$ the estimate has been taken from Schmidt's [2] model computation; this value must be considered quite uncertain. The mass of the halo population II has been obtained from the number of sub-dwarfs in the vicinity of the sun, assuming that the sub-dwarfs and the globular clusters of the halo population have the same distribution over the Galaxy. This estimate is also very uncertain, because we certainly do not know all the sub-dwarfs in our vicinity and therefore this figure represents a lower limit. If the mass values given for the three population groups mentioned are subtracted from Schmidt's estimate of the total mass of the galactic system, there remains $47 \times 10^{9}$ solar masses. Most of this is probably the disk population, with a small contribution from the intermediate population II.

We may imagine the Galactic System to have resulted from the contraction of an irregular mass of gas. This mass of gas may have been endowed with a considerable resultant angular momentum, which will still be largely present in the galactic system. Some of it may have been carried away by escaping stars or masses of gas, but it seems likely that most of it is still present. From estimates of the total angular momentum of the Galactic System we may therefore arrive at an estimate of the total angular momentum that was present in the primeval mass of gas.

The way in which this mass of gas developed would depend in the first 
place on the proportion of regular and irregular motion. If the motions were quite regular this primeval gas would have contracted in a thin disk of approximately constant density. On the other hand, if there was only a small amount of angular momentum present, the primeval gas would have contracted into an almost spherical system. The amount of concentration to its centre would depend on two factors: on the rate of star formation during the various stages of the contraction and on the random motions of the clouds from which the stars were formed. It is evident that the galactic system is something between these two extremes. Its mass has certainly a flattened shape, but at the same time the main mass is strongly concentrated to the centre.

The halo population II, in the first column of Table 2, should then contain the oldest objects, formed before the contraction into the disk. The measure of central concentration of this halo population should thus give important information on the original internal motions in the gas.

It is probable that during the process of contraction to a disk the internal currents of the gas formed layers of high density from which a large number of stars were formed. It is difficult to make an estimate of the time which this contraction will have taken. The age of the halo population II seems to be of the order of $6 \times 10^{9}$ years as derived from the colourmagnitude diagrams of globular clusters. The time of contraction to the disk, during which the intermediate population II was formed, will depend on the character of the regular and irregular streamings that were present in the gas, and on the amount of energy radiated away by the heated hydrogen gas. This heat had to be radiated away in order to make further contraction possible and this puts a limit on the time of contraction. A very rough guess is that it would have taken about $10^{9}$ years for the system to contract from the original stage to the disk stage. After the contraction to the disk had been completed the disk population must have been formed. This is probably the major part of the population of the Galactic System.

Since there must have been a very strong concentration of matter towards the centre by this process, it is not surprising that the disk population exhibits such a concentration, but we may ask why the gas at present shows no concentration at all towards the centre. According to the observations it is rather evenly distributed over the disk, perhaps even somewhat less dense in the central part than in our neighbourhood.

To explain this difference we are probably forced to assume that the star-forming process depends on a fairly high power of the density. If this is so, we may imagine that stars were formed during all stages of the contraction, but that the star-building process was particularly rapid in the 
last stages when matter had become very dense in the inner parts of the disk. In the following, and presumably longest, stage in the existence of the Galactic System star-forming would have gone on more slowly and at a gradually decreasing rate.

If there is any truth in the above sketch of the evolution of the Galactic System the classification given in the table must be roughly a classification by age.

The distinctions in the last columns of Table 2 are of a gradual kind. The very youngest objects and the gas are in the last column, and objects of which we have some indication that they are fairly young have been put in the column next to it. It is difficult to assign ages to objects in the disk population, except in the case of the galactic clusters. The difficulty in disentangling ages of objects in the post-contraction period is due largely to the fact that apparently the dynamical properties of stars formed at different times between 5 and 0.5 billion years ago have changed very little. There are, however, indications that the $\mathrm{O}$ and $\mathrm{B}$ stars and the galactic clusters containing stars of early spectral class have average distances from the galactic plane of only 50 or $60 \mathrm{pc}$, while we find twice these distances for the clusters with ages about $5 \times 10^{9}$ years. This difference may be supposed to be due to the fact that gradually the turbulence of the interstellar gas has been decreasing. It seems, however, more probable that stars and clusters are still being accelerated by encounters with large irregular concentrations of interstellar matter. An ideal way of investigating this last problem would be, as was once tried by Weaver [3], to study galactic clusters, which according to the H-R diagram have different ages, and to see if one can find an increase with age of the average distance from the galactic plane or of the average velocity perpendicular to the plane. There seems to be a good indication that such is the case. One could even from these properties estimate the total effect that encounters with large interstellar masses have had on the velocity distribution.

Some important aspects of the structure of the galactic system are still entirely missing in the sketch given above. It does not explain how the spiral structure, which seems to be such a very common feature of galaxies, has come into existence. The same holds for the bar structure which seems to be so common an element in galaxies.

In recent years more and more evidence has accumulated that stars formed at different epochs have different chemical composition. The fraction of elements heavier than $\mathrm{H}$ and $\mathrm{He}$ appears to increase from about 0.003 in the oldest stars to about 0.04 in the youngest ones. The change is probably connected with a gradual change with time in the 
composition of the interstellar medium from which the stars were formed. If this is so, the enrichment with heavier elements must have been due to transformation of elements inside stars which have subsequently shed a large part of their mass into the interstellar medium again. It is then quite possible that all heavier elements now present in the interstellar medium have been formed in stars. It is still quite uncertain whether the enrichment is due to the explosion of a relatively small number of super-novae or whether the larger part comes from slower processes of expulsion of matter from stars.

Table 2 contains estimates by Schwarzschild [4] of the proportion of elements other than hydrogen and helium in the stars of various population groups. From the fact that some globular clusters at large distances from the galactic plane give evidence of a considerable metal content, while others show hardly any metal lines, and that, as Morgan has pointed out, a number of globular clusters near the galactic nucleus show heavyelement abundances resembling those in the sun, it would appear that the major part of the enrichment with heavier elements has occurred in an early phase of galactic evolution, part of it even before the Galactic System had contracted into a disk.

A development of special interest in the study of galactic structure has recently been given by Salpeter. He remarked that if we suppose that in our vicinity the star-forming process has proceeded at roughly the same rate during the past 4 or $5 \times 10^{9}$ years a plausible form of the generating function (i.e. the luminosity law of stars formed at any given time) would explain the present somewhat peculiar shape of the luminosity function, in particular its rather sudden bend near $M=+3$. The luminosity laws observed in galactic clusters, which resemble Salpeter's generating function, lend support to this idea, as Sandage has shown. This indicates, moreover, that the generating function may have been approximately the same in clusters and in the Galactic System at large. Salpeter has indicated that if this plausible idea of a continuous birth of stars is accepted and if we assume, further, that all the stars brighter than about absolute magnitude +3 , which have burnt up the hydrogen in their cores, have become white dwarfs, the number of white dwarfs in our neighbourhood must be about $10 \%$ of the total number of stars on the main sequence, while in our region of the Galactic System the mass of gas expelled by the stars that are now white dwarfs must have been of the same order as that of all stars or about three or four times that of the interstellar gas now present. This implies that the gas must probably have passed several times through stars.

The appearance of a star is determined largely, if not entirely, by two 
parameters, its mass and its content of heavy elements. If the enrichment with heavy elements has occurred at the same rate in all parts of the Galactic System the heavy-element content of a star would also determine the epoch at which it was formed and, therefore, its age. If this were so and if the heavy-element content of a star could be determined with sufficient accuracy, we might in this way arrange the stars in age groups. In practice we cannot yet reach this ideal. In the first place we can as yet hardly measure the heavy-element content of an individual star except when it deviates drastically from normal, as in the case of the sub-dwarfs. In the second place it is likely that the enrichment of the interstellar material has depended on the amount of star formation and has therefore been much greater in the central parts than in the outer regions.

Attempts to determine the heavy-element content with greater precision must be considered to be of the greatest importance. It is probable, as Sandage has shown, that considerable progress in this line can be made by studying galactic clusters. The main difficulty there is that we know as yet so few clusters of ages intermediate between the primeval and contracting stage on one hand, and the youngest stage of the System on the other hand.

\section{References}

[1] For particulars, see Astronomical Newsletter, no. 71, 10, 1953 and no. 73, 2, 1954 .

[2] Schmidt, M. Bull. Astr. Inst. Netherl. 13, I5, 1956.

[3] Weaver, H. F., unpublished.

[4] See the forthcoming report on the Semaine d'Etude on Stellar Populations, Vatican, 1957, now being edited by $D$. O'Connell.

(B) SOME PROPERTIES OF OTHER STELLAR SYSTEMS

This section deals with a few remarks on stellar systems outside the Galaxy which were considered to be of importance in the interpretation of galactic observations.

\section{(I) Integrated spectra and colours of galaxies}

Information on the general stellar population predominant in the light emitted by galaxies, may be obtained from a study of the integrated spectra. W. W. Morgan reported on such an approach, recently made by Morgan and Mayall [1], extending earlier work by Humason [2] and others.

The criteria used for the spectral classification, which necessarily are of the very low-dispersion variety, are mainly the appearance of the hydrogen lines and the cyanogen absorptions at $\lambda \lambda 3_{3800}$ and 4200 . In objects where the hydrogen lines are of maximum intensity the predominant 